FEDSM2006-98485

\title{
LINEAR STABILITY ANALYSIS OF CHEVRON JET PROFILES
}

\author{
Kristjan Gudmundsson \\ Department of Mechanical Engineering \\ California Institute of Technology \\ Pasadena, California 91106 \\ Email: kristjan@caltech.edu
}

\author{
Tim Colonius \\ Department of Mechanical Engineering \\ California Institute of Technology \\ Pasadena, California 91106 \\ Email: colonius@caltech.edu
}

\begin{abstract}
We investigate the linear stability characteristics of the mean velocity profiles produced by chevron nozzles. We show that chevron instability waves can be decomposed into azimuthal modes analogously to those of round jets. This facilitates a direct comparison of growth rates and mode structure between different nozzles. We find that the three nozzles used in this study share a set of modes, referred to as primary modes. In addition, we find that there exist modes unique to the chevrons nozzles, termed secondary modes. While chevron jets possess a much larger number of unstable modes, the modes with lowest azimuthal structure show strong suppression of growth rates in two different chevron jets. Some preliminary implications on sound generation are discussed.
\end{abstract}

\section{INTRODUCTION}

With turbofan bypass ratios approaching practical limits, different approaches to noise reduction have been pursued, including the machining of serrations, or chevrons, into the nozzle lip. Examples of such nozzles, commonly referred to as chevron nozzles, are shown in Fig. 1. The chevrons generate streamwise vortices that enhance mixing and shorten the potential core. They also reduce noise at low frequencies and aft angles, but increase noise at high frequencies. The high frequency penalty is explained by the increased levels of small scale turbulence, while the noise reduction at lower frequencies, associated with the large scale structures of the flow, is not well understood.

At present, design and deployment of chevron nozzles is based on exhaustive and costly laboratory, and full-scale testing.
Due to the complexity of the near-nozzle flow field, there are currently no physics-based prediction methods for noise reduction; properly resolving the large-scale flow structures is not yet possible, even with modeling tools such as Large Eddy Simulation. As a surrogate, we propose to compute the linear instability modes of the time-averaged chevron flow field, and to investigate the extent to which the chevrons modify the stability characteristics of an equivalent-thrust round jet.

In a strict sense, the concept of a linear instability mode is not applicable to a spatially spreading flow such as a jet (see cartoon in Fig. 2, left). However, such flows can be assumed locally parallel if their spreading rate is negligible over fluctuation length-scales. Under these circumstances, cross sections of the jet, as shown in Fig. 2, right, are analyzed as if each one was a sample from an unbounded parallel mean flow. This approach was taken by [1], where the hydrodynamic pressure field of a round jet was shown to be consistent with that of the instability modes of the spreading mean flow ${ }^{1}$. The appropriateness of the quasi-parallel assumption for the chevron jet is analyzed as follows. The potential core length for the most aggressive chevron we consider is shorter than the round jet by about 2 diameters [2]. This corresponds to an increase in (azimuthally averaged) spread rate from about 0.18 for the straight nozzle to about 0.25 for the chevron nozzle. The PIV data we used in the present study also shows that the azimuthally averaged transverse mean velocity in the chevron jet has a maximum value of about $10 \%$ of the streamwise velocity at $x / D=2$, whereas for the straight nozzle it is $8 \%$. Thus the assumption of locally parallel mean flow is somewhat

\footnotetext{
${ }^{1}$ We note that [1] used the same dataset as this study, for nozzle SMC000 (see Fig. 1).
} 


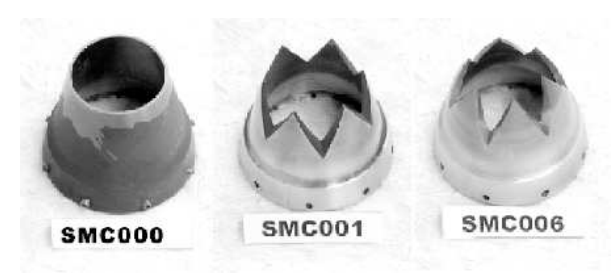

Figure 1. From left: straight nozzle, chevron nozzles with moderate/aggressive penetration.

less appropriate for the chevron jet, but still reasonable as a first step.

Standard quasi-parallel stability analysis of jet flows considers, at each streamwise position, a one-dimensional (Ordinary Differential) eigenvalue problem in the cross flow direction and is amenable to direct matrix solution or shooting algorithms. The chevron jet, on the other hand, is inhomogeneous in both coordinate directions normal to the stream and must be solved using a two-dimensional (Partial Differential) eigenvalue problem. As such it is much more computationally intensive than the onedimensional problem. To solve the chevron stability problem, we have developed an approach that couples a high-order accurate compressible flow solver with the iterative eigensystem solver ARPACK, described in the next sections.

\section{CALCULATION OF INSTABILITY WAVES}

Using far-field sound speed $a_{\infty}$, density $\rho_{\infty}$, and nozzle diameter $D$ for non-dimensionalization, we linearize the flow field about a locally parallel mean flow having axial velocity $\bar{U}(y, z)$, and density $\bar{\rho}(y, z)$, where coordinate configuration is illustrated in Fig. 2. For this purpose, we assume that flow field variables can be decomposed into mean, and fluctuating components, as $u=\bar{U}+u^{\prime}, v=v^{\prime}, w=w^{\prime}, \rho=\bar{\rho}+\rho^{\prime}$, and $p=1 / \gamma+p^{\prime}$, where primed quantities represent small fluctuations, and $\gamma$ is the ratio between specific heats. Substituting these expressions into the Navier-Stokes equations, and only retaining terms first order in fluctuations yields

$$
\frac{\partial \mathbf{q}}{\partial t}+\mathcal{L} \mathbf{q}=\frac{1}{\operatorname{Re}} \mathcal{V} \mathbf{q}
$$

where $\mathbf{q}=\left[(\rho u)^{\prime},(\rho v)^{\prime},(\rho w)^{\prime}, \rho^{\prime}, e^{\prime}\right]^{T}$, Re is the Reynolds number, based on the jet diameter, kinematic viscosity and centerline velocity. $\mathcal{L}$ and $\mathcal{V}$ represent linear differential operators, used here for brevity; the full equations are shown in the appendix. Since we have assumed the mean flow to be locally parallel, the operators $\mathcal{L}$ and $\mathcal{V}$ are homogeneous in the flow direction, so that the solutions $\mathbf{q}$ can be decomposed as

$$
\mathbf{q}(x, y, z, t)=\hat{\mathbf{q}}(y, z) e^{i k x} e^{-i \omega t}
$$

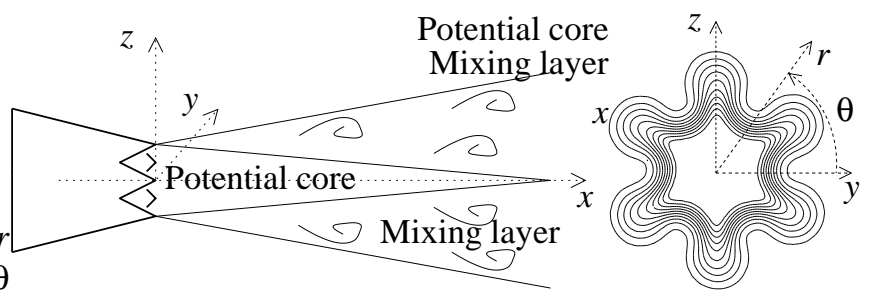

Figure 2. Left: cartoon of jet flow and nozzle. Right: velocity cross section $\hat{U}(y, z)$ of jet from nozzle SMC001 at $x / D=2$.

where $k=k_{r}+i k_{i}$ and $\omega=\omega_{r}+i \omega_{i} . k_{r}$ and $\omega_{r}$ denote the axial wavenumber and frequency, respectively, while $k_{i}$ and $\omega_{i}$ denote amplification rates. If $\omega_{i}>0$ disturbances will grow in time, and if $k_{r}<0$ disturbances will grow in the downstream direction. There are two common approaches to the stability problem; in the temporal approach, one sets $k_{i}=0$, and searches for solutions unstable in time. The alternative is the spatial approach, where one sets $\omega_{i}=0$ and searches for solutions unstable in the flow direction. While the latter has been somewhat more successful in reproducing experimental results, it is also more computationally intensive, as it requires the solution of a generalized eigenvalue problem, rather than a regular one. Furthermore, as the mean flow decays rapidly towards the boundaries of the computational domain, singular terms arise in the governing equations. In the spatial problem, these terms are diagonal, so that the problem becomes ill-posed. We believe that the problem can be regularized, so that eventually one might be able to solve the spatial problem. However, as a first step, we solve the temporal problem.

Substituting Eqn. (2) into Eqn. (1), and letting $\mathcal{A}=\mathcal{L}-$ $\frac{1}{\operatorname{Re}} \mathcal{V}$, we obtain

$$
\mathcal{A} \hat{\mathbf{q}}=i \omega \hat{\mathbf{q}}
$$

an eigenvalue problem. The eigenvectors $\hat{\mathbf{q}}$ represent instability waves having growth rate $\omega_{i}$ and frequency $-\omega_{r}$. The computational procedure for solving the eigenvalue problem is described in the next section.

\section{NUMERICAL METHOD}

Two components are needed in the solution of the eigenvalue problem represented by Eqn. (3). The first is the efficient discretization of $\mathcal{A}$, to obtain $A$, say, and the latter is an algorithm for solving the resulting eigenvalue problem $A \hat{q}=i \omega \hat{q}$, where $\hat{q}$ and $i \omega$ refer to the discrete versions of their continuous analogs. For discretization, we use a compressible flow solver developed and verified by Bres ${ }^{2}$ and Colonius, and used in e.g. [3]. The

\footnotetext{
${ }^{2}$ Guillaume Bres, Graduate student, Dept. of Mechanical Engineering, California Institute of Technology.
} 
code uses sixth-order compact finite differences, with the outflow boundary conditions of [4]. To solve the eigenvalue problem we use the iterative eigenvalue solver ARPACK, which implements the Implicitly Restarted Arnoldi Method, see e.g. [5]. Modes corresponding to specific parts of the eigenspectrum, such as the ones having most negative real part, can be requested. This is essential in our computations, as we look for the most unstable modes, i.e. the ones having largest $\omega_{i}$.

To verify the flow solver-ARPACK combination (hereafter referred to as the solver), the problems of the inviscid instability of a planar shear-layer and a round jet were solved. These problems can be reduced to one-dimensional boundary value problems, solvable with the shooting method. For brevity, we only describe validation with the planar shear-layer. While this case only tests the capabilities of the solver for one-dimensional problems, we note that similar results were found for the round jet, which is a two-dimensional problem as seen by the solver.

Following [6], we use an analytical velocity profile, given by

$$
\bar{U}(y)=\frac{M_{\infty}}{2}\left(1+\tanh \left(2 y / \delta_{\omega}\right)\right),
$$

where $\delta_{\omega}$ is vorticity thickness. Figure 3 shows a comparison of growth rates obtained by the two methods, as a function of wavenumber, with $M_{\infty}=0.8$ and $\delta_{\omega}=1$. As the figure shows, reasonable agreement was obtained, the differences generally being about $1 \%$, in terms of the eigenvalues obtained.

While the shooting code solves the inviscid problem, the solver requires a finite Reynolds number. This is due to the discretization scheme; spurious waves (waves with planar wavenumbers higher than those properly resolved by the scheme) will grow exponentially in time. For a fixed Reynolds number, this can be combated by grid refinement. However, in order to keep the problem size to a minimum, we investigated whether a Reynolds number, or a range of Reynolds numbers, could be found, such that discretization instabilities would be damped, while leaving the growth rates of the physical instabilities relatively unchanged. We found that this was the case, with a Re of order $10^{4}$ being sufficient for the planar shear-layer. This was the value used in the above validation. A similar study was done for the chevron jet, finding again that, beyond $\operatorname{Re} \approx 10^{4}$, physical instabilities seem to be independent of Reynolds number.

\section{DATA PROCESSING}

The data used in this study was obtained from time-averaged PIV measurements conducted by Bridges et al [2], in the Small Hot Jet Acoustic Rig (SHJAR), at the NASA Glenn Research Center. Experiments were performed for several nozzle and flow configurations; more details on the experimental setup can be

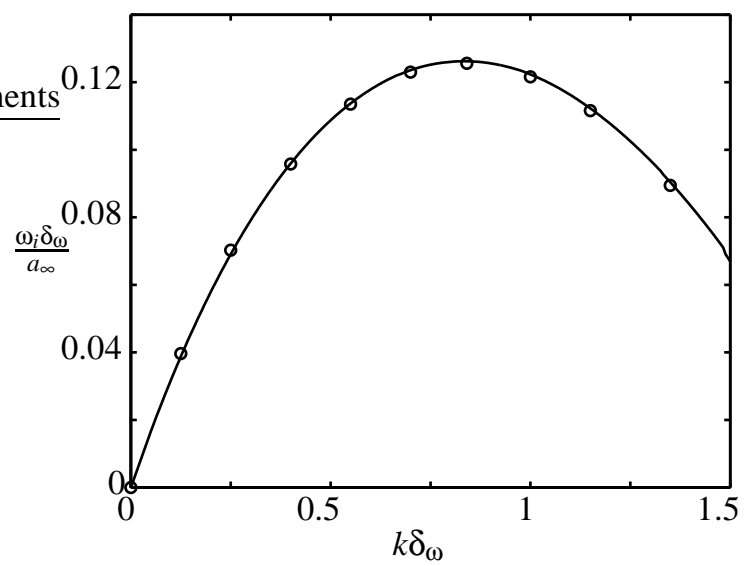

Figure 3. Non-dimensional growth rate $\omega_{i} \delta_{\omega} / a_{\infty}$ vs. non-dimensional wavenumber $k \delta_{\omega}$, for the hyperbolic-tangent velocity profile. Solver solution ( $\circ$ ), shooting solution ( $\longrightarrow$ ).

found in [2]. Our study is limited to cold jets at $M_{\infty}=0.9$, and three thrust-equivalent nozzles, with serial numbers SMC000, SMC 001 and SMC006, depicted in Fig. 1. At this time, we further restrict our attention to data at cross section $x / D=2$.

To assess the instability characteristics of any given jet flow, a computational domain of several jet diameters is needed. As the above PIV data is limited to roughly three jet diameters, an extension is necessary. We do this by smoothly weaving the data with an axisymmetric, exponentially decaying function. We find that the particulars of the extension do not affect the results in a significant way; even setting the data discontinuously to zero will produce reasonable results.

Seeing that the chevron nozzles each have six chevrons, a sixfold symmetry should be observed in the flow field. This is what is seen, but in addition there are components that do not obey this symmetry. This can come about for various reasons, such as slight imperfections in nozzle geometry. We perform an azimuthal Fourier decomposition of the data, $\bar{U}(y, z)$,

$$
\bar{U}(y, z)=\sum_{n=-N / 2}^{N / 2-1} a_{n}(r) e^{i n \theta}
$$

where $r$ and $\theta$ are the radial, and azimuthal coordinates, respectively. We then reconstruct the flow field, using only the Fourier components that obey the expected symmetry,

$$
\hat{U}(y, z)=\sum_{n=-N / 2}^{N / 2-1} a_{6 n}(r) e^{6 i n \theta}
$$

Figure 4 shows the result of this analysis. The filtered velocity profiles, $\hat{U}(y, z)$, were subsequently used in the stability study. 

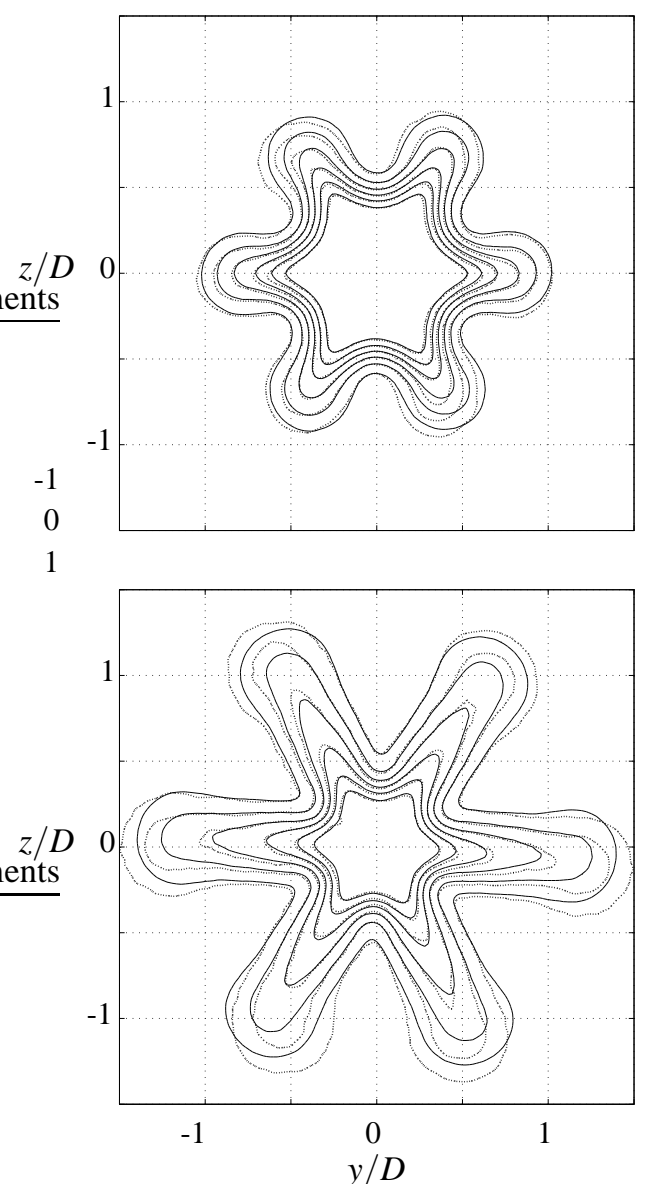

Figure 4. PIV velocity profiles from nozzles SMC001 (top) and SMC 006 (bottom), at $x / D=2$. Unprocessed data $\bar{U}(y, z)(\cdots \cdots \cdot)$, processed data $\hat{U}(y, z)$ ( $)$.

We stress that the filtering does not significantly alter the instability characteristics of the flow field (such as the growth rates), but results in a significant reduction in the number of iterations needed for convergence, as well as a greater symmetry between eigenmode pairs (see next section).

\section{RESULTS AND DISCUSSION Classification of Chevron Instability Waves}

We have discovered that chevron instability waves have several characteristics in common with those of round nozzles. As the latter serve as the basis for comparison for the more complex chevron case, let us review some of their basic properties. Despite the results being computed on a rectangular mesh, they are most conveniently interpreted in a cylindrical coordinate system, shown in Fig. 2, right.

As previously mentioned, the problem of the inviscid instability of a round jet can be reduced to a one-dimensional bound- ary value problem. This is done by assuming the pressure instability wave, $P(r, \theta)$, to have the form

$$
P(r, \theta)=\hat{P}(r) e^{ \pm i m \theta}
$$

where $m$ is the azimuthal wavenumber. Note that since the round jet is axisymmetric, any rotation of $P$ will again be an eigenvector. Such rotated vectors, $P(r, \theta+\phi)$, can be obtained by a phase shift of the original vector through $P(r, \theta+\phi)=e^{i m \phi} P(r, \theta)$.

Upon substitution of Eqn. (7) into the inviscid Navier-Stokes equations in cylindrical form, they collapse to a single ordinary differential equation for $\hat{P}(r)$. This ODE only depends on $m$ through $m^{2}$, so that we obtain two equivalent solutions, for $\pm m$, aside from when $m=0$. The physical significance of the positive/negative modes can be seen from the azimuthal momentum equation, which reduces to

$$
u_{\theta}=\frac{i}{k \bar{U}-\omega} \frac{\partial P}{\partial \theta}=\frac{ \pm m P}{k \bar{U}-\omega}
$$

Thus, the positive/negative modes signify flow swirling in opposite azimuthal directions.

Our results indicate that most chevron instability modes also come in pairs, much akin to those described above. However, the chevron jet has a different symmetry; only rotations of eigenvectors by 60 degrees are permissible, assuming six chevrons. In addition, all six rotations are equally valid solutions. Thus, we must be able to construct them all from the one that does come out of the solver, $P(r, \theta)$, say. In other words, $P(r, \theta)$ forms a basis for the subspace to which the six rotations belong, so that we obtain different rotations $P(r, \theta+\phi)$, through a linear combination (of a single vector),

$$
P(r, \theta+\phi)=e^{i \gamma} P(r, \theta)
$$

where $\phi$ is any integer multiple of $2 \pi / 6$. This suggests that $P(r, \theta)$ can be decomposed as

$$
P(r, \theta)=\hat{P}(r, \theta) e^{ \pm i m \theta}
$$

where $\hat{P}(r, \theta+\phi)= \pm \hat{P}(r, \theta)$. Note that Eqn. (10) has similar form as Eqn. (7); the difference lies in the more complicated amplitude function $\hat{P}(r, \theta)$. Expanding this function in azimuthal Fourier modes, and applying the constraint $\hat{P}(r, \theta+\phi)=$ $\pm \hat{P}(r, \theta)$, we see that, for six chevrons, we must have

$$
P(r, \theta)=\left[\sum_{n=-N / 2}^{N / 2-1} a_{3 n}(r) e^{3 i n \theta}\right] e^{ \pm i m \theta}
$$


where $N$ is a sufficiently large integer.

Similarly to the round jet, we get modes corresponding to $m=0,1$ etc. However, for the chevron jet, we get more than one mode corresponding to $m=0$, as well as more than one pair of modes corresponding to $m=1$ or 2 , where the multiple pairs have different amplitude $(\hat{P}(r, \theta))$ functions, and different eigenvalues $\omega$. In this way, the chevron jet has many more unstable modes than the thrust-equivalent round jet.

For the chevron jet, one pair of modes for each non-zero $m$ has a phase speed comparable to the corresponding (having the same $m$ ) pair of the round jet. We term this the primary mode. Additional pairs have significantly lower phase speeds and/or growth rates. We refer to these modes as secondary modes. This division is illustrated in Fig. 5, which shows a scatter-plot of the eigenvalues corresponding to the fifteen most unstable modes returned by the solver, using nozzle SMC001 at $k D=2.06$ and $x / D=2$. Figure 6 shows eigenvectors $P$ and amplitude functions $\hat{P}$, for the primary mode, and a secondary mode, for $m=1$ and nozzle SMC001. The primary modes seem to be modifications to the corresponding modes of the round jet, while the secondary modes are unique to the chevron nozzle.

In the case of $m=0$, we have two modes with comparable phase speeds, but different growth rates. These are in fact both comparable to the round jet $m=0$ mode; one having $\hat{P}(r, \theta+2 \pi / 6)=\hat{P}(r, \theta)$, and the other $\hat{P}(r, \theta+2 \pi / 6)=-\hat{P}(r, \theta)$. The latter has a somewhat lower growth rate; we use the former mode to represent the pair (see next section). We note that modes corresponding to $m>2$ were found to be considerably more stable than both the primary and secondary modes for $m=0$ through 2.

Note that the physical significance of the positive/negative modes is altered from that of Eqn. (8), where $\pm m$ signified flow swirling in opposite azimuthal directions. For the chevron jet, we have

$$
u_{\theta}=\frac{i}{k \bar{U}-\omega}\left[\frac{1}{\hat{P}} \frac{\partial \hat{P}}{\partial \theta} \pm i m\right] P
$$

so that it seems chevrons offset the swirling flow of the round jet to a non-zero mean value, which is consistent with the chevrons promoting mixing, above and beyond that of the round jet.

\section{Instability Growth Rates and Phase Speeds}

Figures 7,8 and 9 show non-dimensional growth rate $\omega_{i} D / a_{\infty}$ and phase speed $c_{g}=-\omega_{r} /\left(k a_{\infty}\right)$ vs. wavenumber $k D$, for primary chevron modes with azimuthal mode numbers $m=0$, 1 and 2 , respectively.

Perhaps the most striking feature of these results is the degree to which the chevron jet growth rates are damped, as compared to those of the thrust-equivalent round jet. A natural question to ask is whether the lower growth rates can be attributed

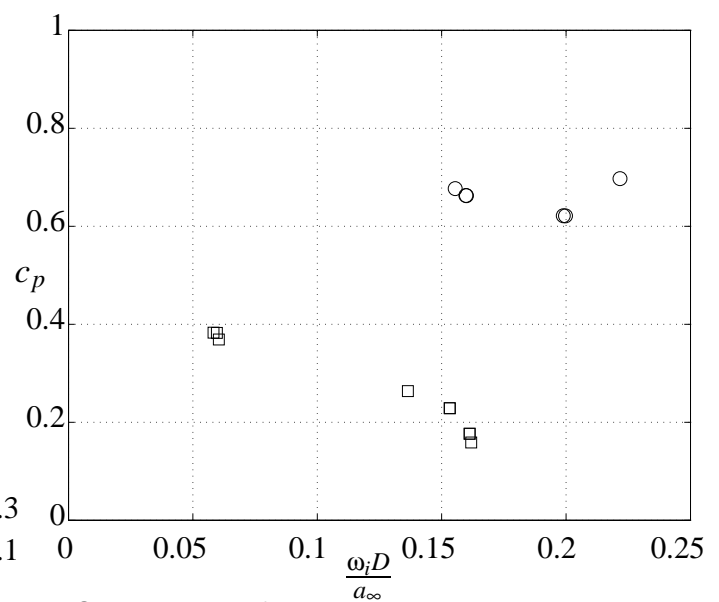

Figure 5. Scatter-plot of non-dimensional phase speed $c_{p}=$ $-\omega_{r} /\left(k a_{\infty}\right)$ vs. non-dimensional growth rate $\omega_{i} D / a_{\infty}$, at nondimensional wavenumber $k D=2.06$, and axial location $x / D=2$. Points correspond to fifteen most unstable modes returned by the solver. Modes identified as primary ( $\circ)$, and secondary $(\square)$.

to chevrons jets having, on average, a thicker shear-layer than the round jet. To estimate this, the linear stability modes of the round jets obtained by azimuthally averaging the chevron jets were calculated. The results clearly show that chevrons provide a mechanism that not only damps the growth rates further than the azimuthally averaged profiles would indicate, but also shifts the maximum growth rate towards lower wavenumbers, which signals a degraded acoustic efficiency.

Figure 10 shows a comparison between the primary mode and example secondary modes corresponding to $m=0$. These results seem to indicate that the secondary modes have a different characteristic length-scale than the primary modes. In particular, it seems the secondary length-scale is considerably smaller than the primary one; this is evidenced by the shift towards higher wavenumbers. Such a division could potentially be obtained by the primary length-scale being the jet diameter, and the secondary length-scale being the width of the chevron fingers.

Sound radiated from jets at shallow angles and low frequency has typically been associated with large-scale structures that are in turn related to instabilities of the inflection mean velocity profile $[7,8]$. Qualitative [9-11] and quantitative analysis [12] connect the envelope and phase speed of instability waves with the acoustic efficiency and directivity at these aft angles. Here we do not attempt any detailed comparison between the instability results and acoustic far field for the chevron jets. However, some observations based on the data are appropriate. First, we observe a suppression of growth rate for the modes we identify as the primary modes for $m=0,1$ and 2 that is consistent with low frequency noise reductions in the three nozzles. 

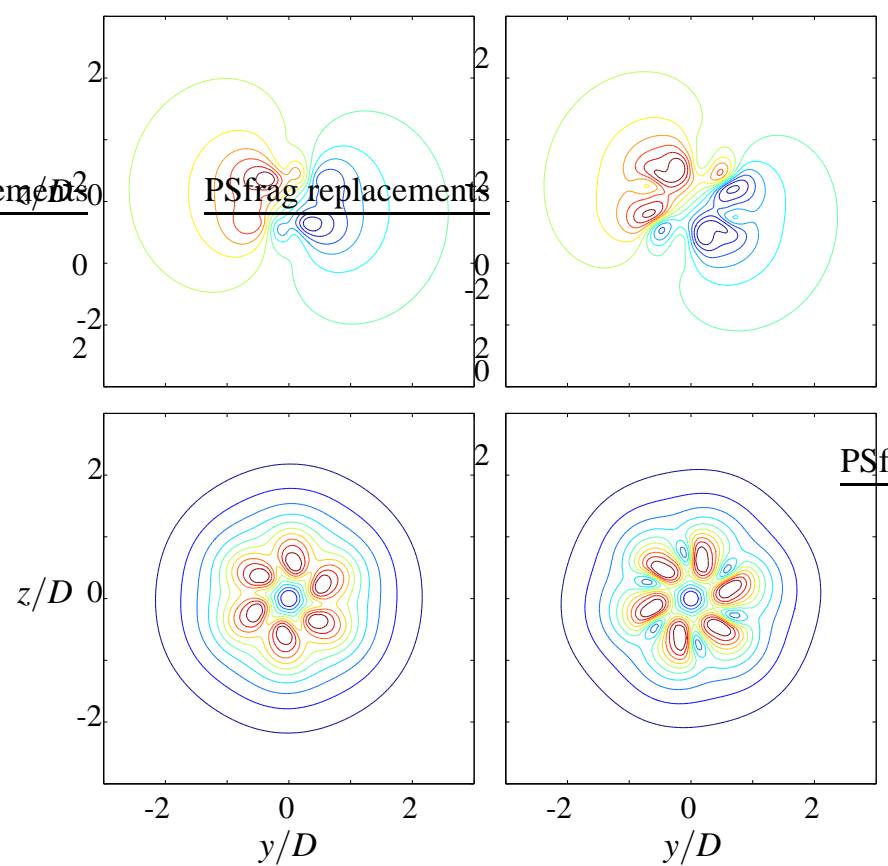

Figure 6. Top: Primary (left) and secondary (right) eigenvectors $P(r, \theta)$. Bottom: corresponding amplitude functions $\hat{P}(r, \theta)$. Nozzle SMC001 with $m=1, k D=1$ and $x / D=2$.

That is, the high penetration chevrons that have the highest low frequency noise suppression also have the lowest growth rates. Quantitatively, for example, for $m=0$ at a fixed wavelength in the temporal analysis that corresponds to the most amplified wave at $x / D=2$, the growth rate is suppressed by about $85 \%$ compared to the circular nozzle.

Another important aspect of the acoustic efficiency of wave packets is their phase speed: the closer to sonic (with respect to ambient), the higher the radiation. However, the instability waves that we identify as primary modes in the chevron jets have phase speeds that increase by as much as about $25 \%$ compared to the circular nozzle. While a firm conclusion awaits a more detailed analysis, it would appear that the growth rate suppression is the more significant effect. Finally, we note that while there are a large number of secondary unstable modes in the chevron jets with comparable growth rates to the primary modes, their phase speeds are low, less than $50 \%$ of the ambient sound speed, and are thus unlikely to contribute significantly to the radiated sound.

We note that the above results have been obtained from computations at axial station $x / D=2$, and as such are limited in scope. However, we have performed a number of computations at other axial stations. While sparse, this additional data is consistent with that of the present study. There is also a question of whether the nozzles should be compared at the locations corresponding to their peak emission frequencies, rather than at the
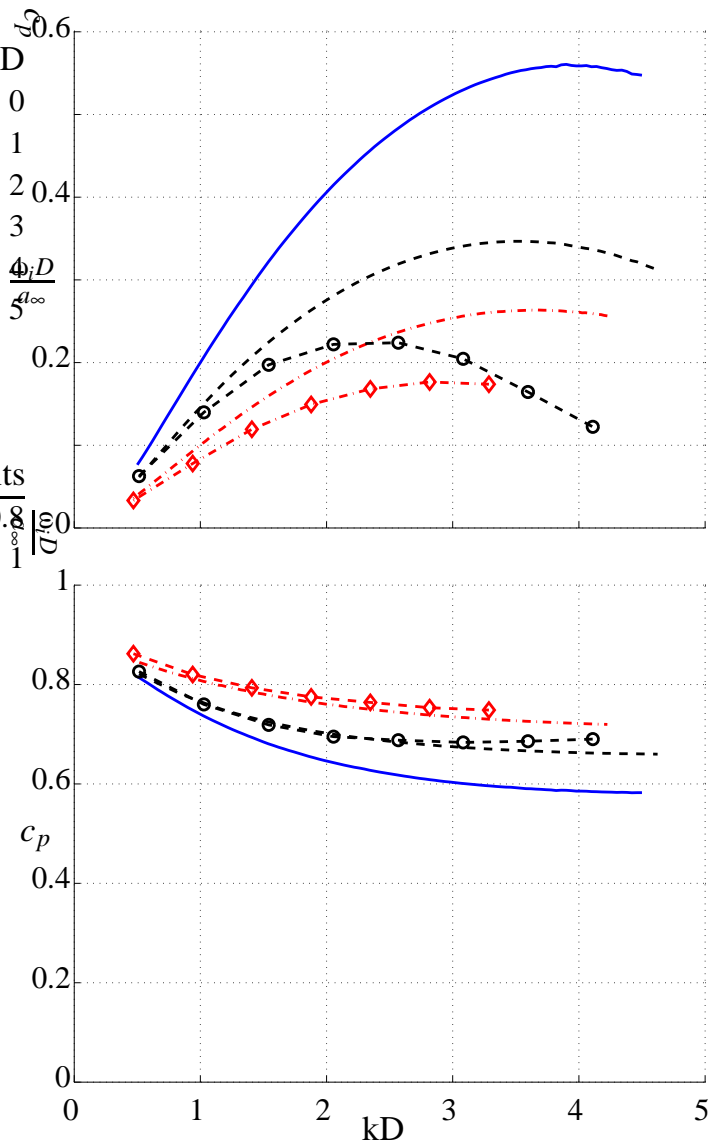

Figure 7. Non-dimensional growth rates $\omega_{i} D / a_{\infty}$ (top) and phase speeds $c_{p}=-\omega_{r} /\left(k a_{\infty}\right)$ (bottom) vs. non-dimensional wavenumber $k D$, for $m=0$ and $x / D=2$. Nozzle $\operatorname{SMC000}(-)$ ). Nozzle SMC0 01 : azimuthal average (---- ) and primary mode(---- 0 ). Nozzle SMC006: azimuthal average ( - - - ) and primary mode ( $-\cdot-\diamond)$.

same downstream location, $x / D=2$ in this case. This will be addressed by more extensive computations, over a wider range of axial stations.

Further, as mentioned above, the spatial approach to the stability problem has enjoyed greater success in explaining experimental results. We will examine the feasibility of solving the spatial stability problem for the chevron jet.

\section{ACKNOWLEDGMENTS}

The authors acknowledge the support of an AearoAcoustics Research Consortium (AARC) grant from the Ohio Aerospace Institute (OAI). In addition, we would like to express great appreciation to Dr. James Bridges and colleagues at the NASA Glenn Research Center for collecting and providing the data used in this study. In addition, we would like to thank Dr. Takao Suzuki for 

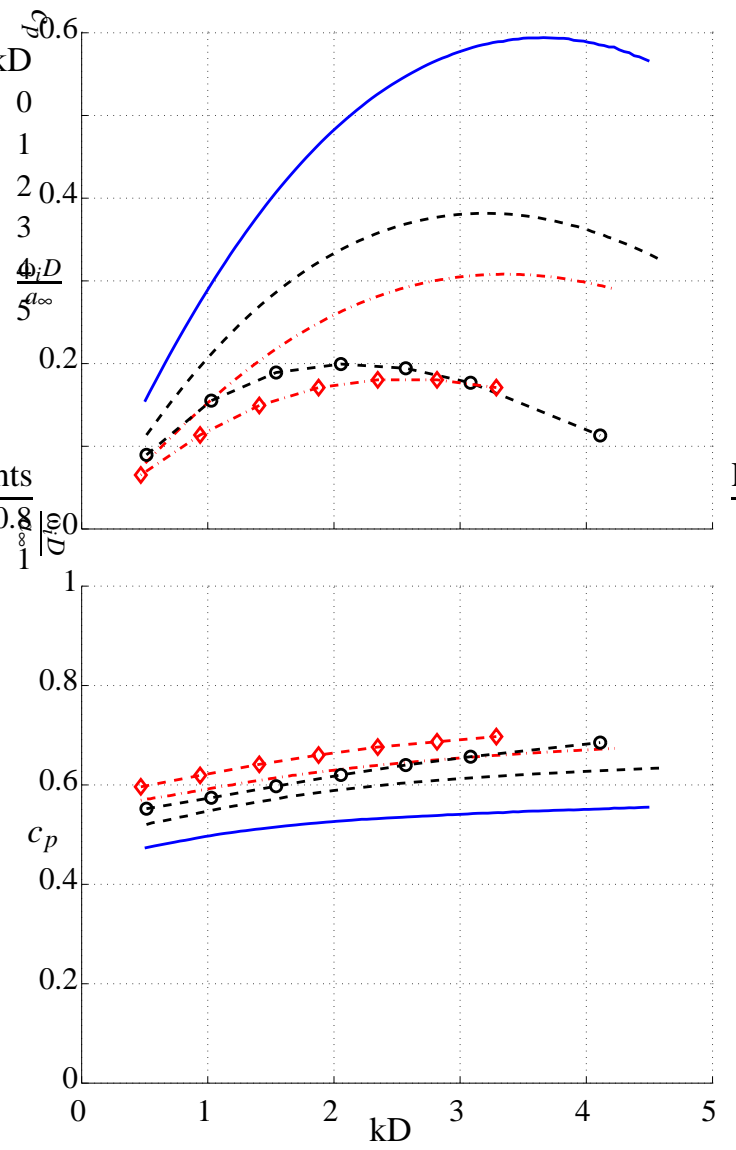

Figure 8. Non-dimensional growth rates $\omega_{i} D / a_{\infty}$ (top) and phase speeds $c_{p}=-\omega_{r} /\left(k a_{\infty}\right)$ (bottom) vs. non-dimensional wavenumber $k D$, for $m=1$ and $x / D=2$. Notation is the same as in Fig. 7 .

useful discussions.

\section{Appendix: Equations used in computations}

Using tensorial notation, we decompose the conserved variables as

$$
\begin{aligned}
(\rho u)_{i} & ={\overline{(\rho u)_{i}}}+(\rho u)_{i}^{\prime}, \\
\rho & =\bar{\rho}+\rho^{\prime}, \\
e & =\bar{e}+e^{\prime},
\end{aligned}
$$

for $i=1,2,3$ (corresponding to $x, y$ and $z$, respectively), where bars denotes base states and primes denote fluctuations. Using this notation, the linearized continuity equation is

$$
\frac{\partial \rho^{\prime}}{\partial t}+\frac{\partial}{\partial x_{j}}(\rho u)_{j}^{\prime}=0
$$
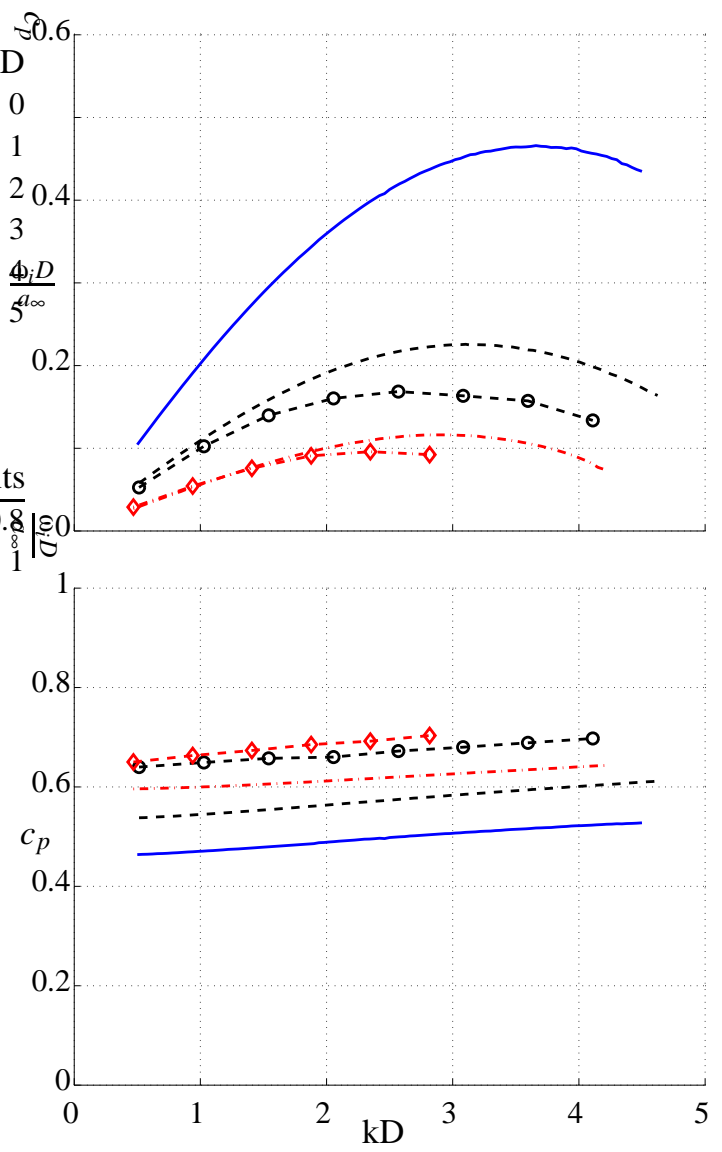

Figure 9. Non-dimensional growth rates $\omega_{i} D / a_{\infty}$ (top) and phase speeds $c_{p}=-\omega_{r} /\left(k a_{\infty}\right)$ (bottom) vs. non-dimensional wavenumber $k D$, for $m=2$ and $x / D=2$. Notation is the same as in Fig. 7 .

the momentum equations,

$$
\begin{array}{r}
\frac{\partial(\rho u)_{i}}{\partial t}+\frac{\partial}{\partial x_{j}}\left(\overline{(\rho u)_{i}} u_{j}^{\prime}+(\rho u)_{i}^{\prime} \bar{u}_{j}+p^{\prime} \delta_{i j}\right)= \\
\frac{1}{\operatorname{Re}} \frac{\partial}{\partial x_{j}}\left(\frac{\partial u_{i}^{\prime}}{\partial x_{j}}+\frac{\partial u_{j}^{\prime}}{\partial x_{i}}-\frac{2}{3} \frac{\partial u_{k}^{\prime}}{\partial x_{k}} \delta_{i j}+\frac{\partial \bar{u}_{i}}{\partial x_{j}}\right)
\end{array}
$$

and the energy equation,

$$
\begin{array}{r}
\frac{\partial e^{\prime}}{\partial t}+\frac{\partial}{\partial x_{j}}\left((\bar{e}+\bar{p}) u_{j}^{\prime}+\left(e^{\prime}+p^{\prime}\right) \bar{u}_{j}\right)= \\
\frac{1}{\operatorname{Re}} \frac{\partial}{\partial x_{j}}\left(\bar{u}_{i}\left(\frac{\partial u_{i}^{\prime}}{\partial x_{j}}+\frac{\partial u_{j}^{\prime}}{\partial x_{i}}-\frac{2}{3} \frac{\partial u_{k}^{\prime}}{\partial x_{k}} \delta_{i j}+\frac{\partial \bar{u}_{i}}{\partial x_{j}}\right)\right)+ \\
\frac{1}{\operatorname{Re}} \frac{\partial}{\partial x_{j}}\left(u_{i}^{\prime}\left(\frac{\partial \bar{u}_{i}}{\partial x_{j}}+\frac{\partial \bar{u}_{j}}{\partial x_{i}}\right)\right)+\frac{1}{\operatorname{RePr}} \frac{\partial^{2} T^{\prime}}{\partial x_{k} \partial x_{k}}
\end{array}
$$



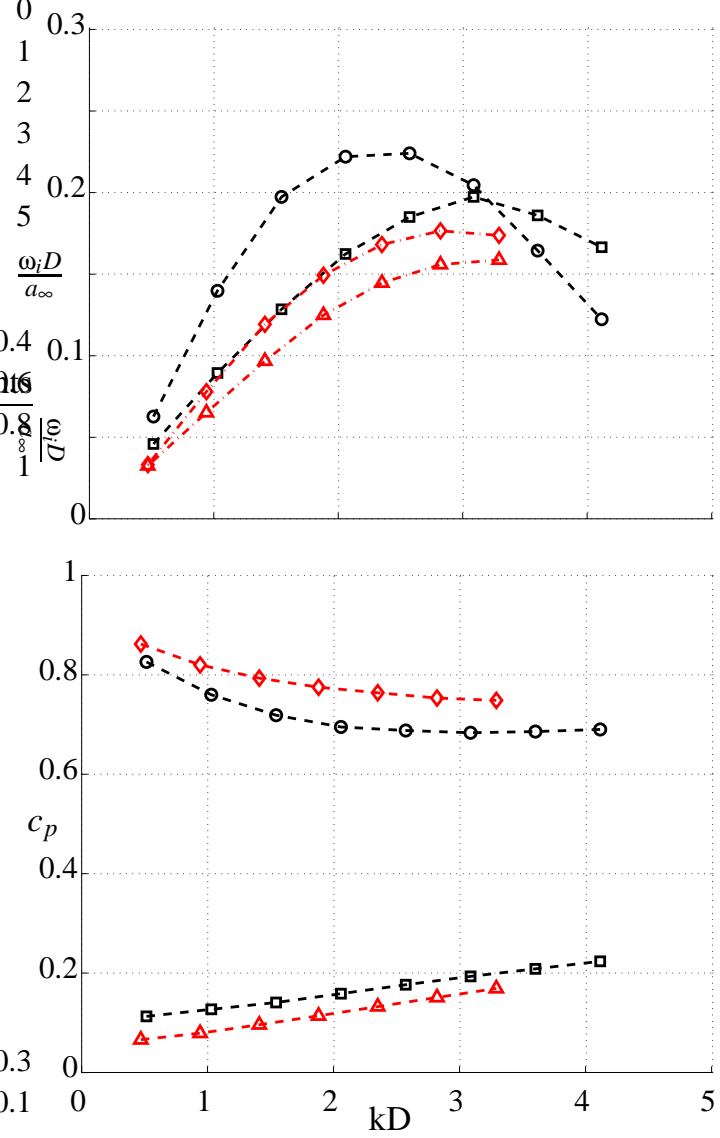

Figure 10. Non-dimensional growth rates $\omega_{i} D / a_{\infty}$ (top) and phase speeds $c_{p}=-\omega_{r} /\left(k a_{\infty}\right)$ (bottom) vs. non-dimensional wavenumber $k D$, for $m=0$ and $x / D=2$. Nozzle SMC001: primary mode ( ---- $\circ$ ), secondary mode (---- $\square$ ). Nozzle SMC006: primary mode $(-\cdot-\diamond)$, secondary mode $(-\cdot-\triangle)$.

where we obtain $u_{i}^{\prime}$ through

$$
u_{i}^{\prime}=\frac{1}{\bar{\rho}}\left((\rho u)_{i}^{\prime}-\rho^{\prime} \bar{u}_{i}\right)
$$

To obtain $p^{\prime}$, we use the definition of the energy $e$,

$$
e=\frac{p}{\gamma}+\frac{1}{2} \rho u_{i} u_{i}
$$

which we linearize, obtaining

$$
p^{\prime}=\gamma\left(e^{\prime}-\frac{1}{2}\left(\overline{(\rho u}_{i} u_{i}^{\prime}+(\rho u)_{i}^{\prime} \bar{u}_{i}\right)\right)
$$

Lastly, we linearize the equation of state,

$$
T=\frac{\gamma}{\gamma-1} \frac{p}{\rho}
$$

obtaining

$$
T^{\prime}=\frac{\gamma}{\gamma-1}\left(\frac{p^{\prime}}{\bar{\rho}}-\frac{\bar{p} \rho^{\prime}}{\bar{\rho}^{2}}\right)
$$

\section{REFERENCES}

[1] Suzuki, T., and Colonius, T., 2006. "Instability waves in a subsonic round jet detected using a near-field phased microphone array". J. Fluid Mech., In Press.

[2] Bridges, J., and Brown, C. A., 2004. "Parametric testing of chevrons on single flow hot jets". AIAA J., 2004-2824.

[3] Theofilis, V., and Colonius, T., 2004. "Biglobal instabilities of $2 \mathrm{~d}$ compressible and model $3 \mathrm{~d}$ incompressible flow over open cavities". AIAA J., 2004-2544.

[4] Thompson, K. W., 1990. "Time-dependent boundary conditions for hyperbolic systems, II". J. Comput. Phys., 89, pp. 439-461.

[5] Lehoucq, R., and Sorensen, D. C., 1996. "Deflation techniques for an implicitly restarted arnoldi iteration". SIAM J. Mat. Anal. and Appl., 17(4), pp. 789-821.

[6] Michalke, A., 1964. "On the inviscid instability of the hyperbolic-tangent velocity profile". J. Fluid Mech., 19(4).

[7] Crighton, D. G., and Gaster, M., 1976. "Stability of slowly diverging jet flow”. J. Fluid Mech., 77, pp. 397-413.

[8] Mankbadi, R., and Liu, J. T. C., 1981. "A study of the interactions between large-scale coherent structures and finegrained turbulence in a round jet". Proc. Roy. Soc. London, 1443, pp. 541-602.

[9] Crighton, D. G., and Huerre, P., 1990. "Shear-layer pressure-fluctuations and superdirective acoustic sources". J. Fluid Mech., 220, pp. 355-368.

[10] T. Colonius, S. K. L., and Moin, P., 1997. "Sound generation in a mixing layer". J. Fluid Mech., 330, pp. 375-409.

[11] Freund, J., 2001. "Noise sources in a low-reynolds-number turbulent jet at mach 0.9". J. Fluid Mech., 438, pp. 277305.

[12] R. Reba, S. Narayanan, T. C., and Dunlop, M., 2003. "A study of the role of organized structures in jet noise generation". AIAA Paper 2003-3314. 